\title{
THE APPLICATION OF THE INTEGRATED INDICATORS BASED ON BP NEURAL NETWORK IN COLLIERY EQUIPMENT SAFETY MONITORING
}

\author{
SHU-FANG ZHAO, LI-CHAO CHEN \\ Institute of Computer Science and Technology, Taiyuan University of Science and Technology, \\ Taiyuan, Shanxi 030024, China. \\ E-mail: zhaoshufang813@sina.com
}

\begin{abstract}
For the current problems in the security situation of colliery equipment, and based on non-linear relationship among the parameters of colliery equipment, this paper presents a method for forecasting the safety of colliery equipment based on BP neural network. By using BP neural network in the colliery safety equipment monitoring and warning issues, we established a multi-index comprehensive monitoring (evaluation) of the device security early warning network model, and have trained and tested the model which used the measured data as an example. The results show that the network performance good and the prediction accuracy is high and user-friendly. So we believe it is an effective method for safe comprehensive evaluation.
\end{abstract}

Keywords: colliery equipment Safety Monitoring

BP neural network $\quad$ Warning

\section{INTRODUCTION}

Colliery equipment to be in motion in safe and reliable status is the basic requirements of colliery enterprises to achieve the nature of the security, and to improve colliery product safety monitoring is an important aspect of technology equipment support system. Colliery equipment condition monitoring is to monitor the right operation of the device as a whole or the state of the key components which can affect the normal operation of equipment, to determine its functioning is normal, to track the status of abnormal, and to forecast trends in abnormal state. It is an important tool and basis to ensure that the safety performance of colliery equipment and colliery safety supervision.Through the monitoring of equipment condition to transformate the risk from prevention after to advance. From the colliery safety analysis concerning the cause of the accident, in which a large part of the accident was mainly due to aging or obsolete equipment, lack of maintenance, security protection facilities, incomplete, improper operation, simplify and neglect routine maintenance equipment, maintenance, parts failures and accidents resulting failure hidden, and induce mechanical damage, fire, gas explosions and other serious accidents. Therefore, from the start with colliery equipment to enhance the testing and management of the safe operation of colliery equipment is one of the primary tasks of the effective exclusion of colliery equipment operation safety hazards and it can 
ensure safety in colliery.

$\mathrm{BP}$ artificial neural network is a nonlinear dynamic system with self-organization, self-learning ability, and it has a strong non-linear fault-tolerance.The BP neural network used in colliery safety equipment forecast allows the data with a strong noise, which is other methods can not be matched. Therefore, this paper presents a forecasting method which is based on BP neural network for colliery safety equipment.

\section{BP NEURAL NETWORK AND MODEL CONSTRUCTION}

Colliery equipment safety monitoring is a complex multi-index nonlinear system, and the indicators are interrelated, which requires monitoring system has some intelligent information processing capabilities in order to ensure the reliability of monitoring. Artificial neural network simulation of human brain activity has a strong non-linear mapping, large-scale parallel processing capabilities, as well as adaptive, self-training, learning, self-organization and fault tolerance, etc., and it develops a new way for the research such as the pattern recognition, non-linear classification, artificial intelligence, etc..

BP neural network model consists of input layer, output layer and one or several hidden layer structures, each layer contains a number of neurons, and layer and layer neuron by connecting the weight threshold interconnects. Theory proved that with a hidden layer of three-layer BP network can approximate any rational function.

The colliery safety monitoring equipment, are as shown in Figure 1, the three-tier structure of BP neural network, namely an input layer, one hidden layer and an output layer, to achieve an arbitrary M-dimensional to the $\mathrm{N}$-dimensional mapping; to BP artificial neural network technology for colliery safety monitoring equipment take unique advantage of the aspects of problem solving in the use of non-linear problems or non-structural ones.

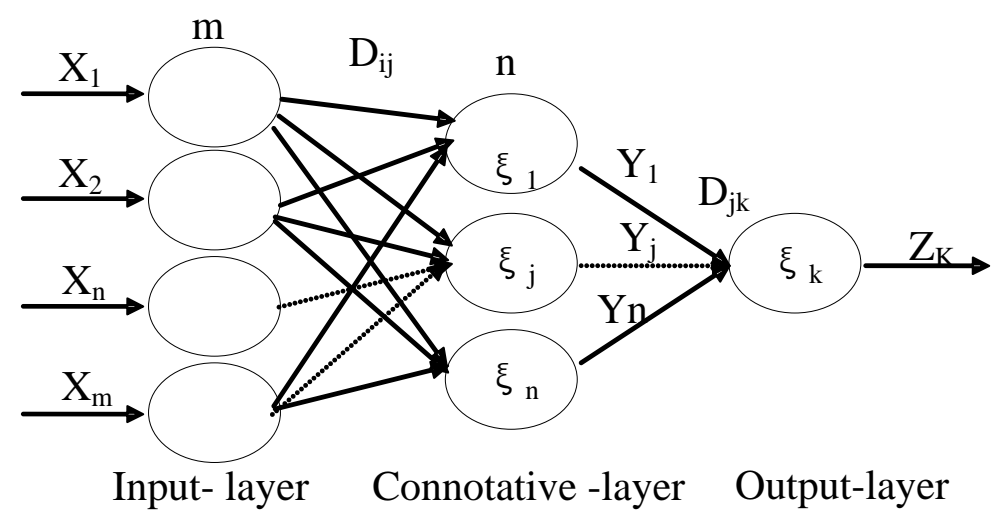

Figure 1 Three-layer BP neural network model

Figure 1, the input layer has $\mathrm{m}$ nodes, input vector $\mathrm{X} \in \mathrm{R}^{\mathrm{m}}, \mathrm{X}=\left(\mathrm{X}_{1}, \mathrm{X}_{2}, \ldots, \mathrm{X}_{\mathrm{m}}\right)^{\mathrm{T}} ; 1 \leqslant \mathrm{i} \leqslant$ $\mathrm{m}$; hidden layer has $\mathrm{n}$ nodes, hidden layer output value $Y \in R^{n}, Y=\left(Y_{1}, Y_{2}, \ldots, \quad Y_{n}\right)^{T} ;, 1 \leqslant j$ $\leqslant \mathrm{n}$; The output layer has $\mathrm{k}$ nodes, the output layer the first $\mathrm{i}$ input layer neurons to the hidden layer

output value $\mathrm{Z} \in \mathrm{Rk}, \mathrm{Z}=\left(\mathrm{Z}_{1}, \mathrm{Z}_{2}, \ldots, \mathrm{Z}_{\mathrm{k}}\right)^{\mathrm{T}}$, in Figure $1 \mathrm{k}=1$. Input layer and hidden layer have connection weights between the value of $D_{i j} . D_{i j}$ denotes that 
neuron j-month weight, the Threshold is $\xi \mathrm{j}$. The hidden layer and output layer have connection weights between the value of $D_{j k}$, the Threshold is $\xi_{\mathrm{k}}$; Hidden layer and output layer output values to meet the : $Y_{j}=f\left(\sum_{i=1}^{m} D_{i j} X_{i}-\xi_{j}\right) ; Z_{k}=f$ $\left(\sum_{j=1}^{n} D_{j k} Y_{j}-\xi_{k}\right)$

Function as the activation function $f$, Meet: $f(x)$ $=\frac{1}{1+e^{-x}}$

Set $D_{j k}$ the connection weights between hidden layer nodes and the output layer nodes in the moment of connection , $D_{i j}$ is the connection weights between hidden layer nodes and the input layer nodes in the moment of connection, $\eta$ $(0<\eta<1)$ is a positive constant term, called the learning rate, which reflects the adjustment of rates of weight. If $\eta$ is too small, the lower the efficiency of learning is, the convergence speed is much more slower; Conversely, if too large, skipped the optimal value point, it may cause oscillation. To this end, the introduction of momentum term $\alpha(0<\alpha<1)$ can filter out the weight room in the high-frequency deviation of the error surface, making the learning process is equivalent to change the value $\eta$, so adjust the direction of change towards the bottom of the average will not have a big swing that the momentum role cushion and smoothing effect.ion.

Under normal circumstances, momentum term can reduce the oscillation, at the same time, the algorithm converges faster. In the three-layer BP neural network, the greatest impact on network performance weights is Amendment Act, this paper adopts the following method to amend the value of:

$$
\begin{gathered}
D_{j k}(N+1)=D_{j k}(N)-\eta \frac{\partial E}{\partial D_{j k}}+\alpha\left(D_{j k}(N)-D_{j k}(N-1)\right) \\
D_{i j}(N+1)=D_{i j}(N)-\eta \frac{\partial E}{\partial D_{i j}}+\alpha\left(D_{i j}(N)-D_{i j}(N-1)\right)
\end{gathered}
$$

Where $\alpha$ is the impulse coefficient, generally taking about 0.7 .

\section{SAFETY EQUIPMENT FORECAST}

Colliery safety equipment is to predict device status information based on the past to predict the device's security status.To make the right security decisions about the entire colliery depend primarily on the safety of colliery equipment to make accurate forecasts, accurate prediction is to take the right management programs and control measures as a prerequisite. Equipment condition monitoring is designed to determine the state of the machine running, once it is abnormal, alarm or stop power outage. In order to accurately grasp the state of the equipment basic information, some of the parameters must be monitored. Monitoring equipment status parameter is a reflection of equipment and a necessary condition for fault diagnosis.

The system uses object-oriented design methods and WEB modeling methods. The method of describing mainly includes relationship diagram and timing diagram between the WEB page objects and so on. The sequence diagram denotes the time-based dynamic interaction between the objects. It is expressed visually how objects interact over time. Object icons arrange in the top of the sequence diagram and use solid lines with arrows to denote the message as well as use the vertical dotted line represents time. That is the arrangement of 
objects from left to right (on behalf of the layout of the object dimensions), and use solid lines with arrows to denote the message passing; From up to down is time passing (on behalf of the time dimension). Among them, the message can be simple (Simple), synchronous (Synchronous) or asynchronous (Asynchronous).

\subsection{Predictors of Colliery Safety Equipment}

Colliery equipment for reliable operation is essential to ensure the colliery safe production. This article divides the colliery equipment into six major categories of colliery equipment, and then each category of equipment extracts the key indicators that affect the equipment safety. Its hierarchical structure is shown in Figure 2:

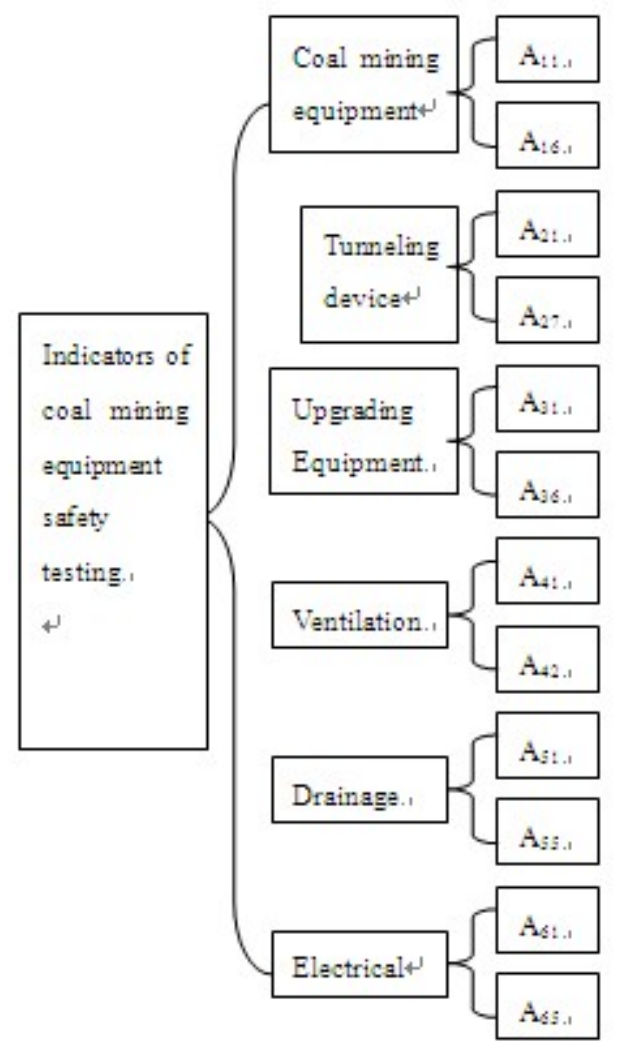

Figure 2 device status monitoring indicators

(1)Coal mining equipment. Coal mining equipment is the major colliery production safety equipment. Equipment condition monitoring indicators include six key indicators: Hydraulic support (A11),Shearer (A12),Scraper conveyor (A13),Crusher (A14) etc..

(2)Tunneling device. Tunneling device is mainly used to excavate mine tunnel. Equipment condition monitoring indicators include seven key indicators: TBM ( A21) ,Hydraulic Drill (A22, Explosion-proof air compressor(A23),etc..

(3)Upgrading Equipment. Upgrading equipment is the major colliery equipment for lifting people and materials. Equipment condition monitoring indicators include six key indicators : Hoist(A31),Cage(A32),Reducer table (A33), etc..

(4)Ventilation. Ventilation equipment is to transport the air underground, diluting and expelling harmful gases from underground to ensure that underground workers is in sufficient quantity to meet the requirements for breathing air. Equipment condition monitoring indicators include two main key indicators: fanner(A41),And the duct (A42) .

(5)Drainage. Colliery drainage is one of the large-scale equipment; its efficiency will directly affect the level of the economic benefits of colliery enterprises. Equipment condition monitoring indicators include five key indicators: Pump (A51), Auxiliary motor(A52), Water-level alarm(A53),etc..

(6)Electrical Equipment. Colliery electrical equipment throughout the colliery safety management is an important component of safety management. To strengthen and improve the electrical equipment safety management, this system is one of good guarantees of the job of colliery safety and it has a very important role in avoiding and reducing the occurrence of major accidents. Equipment condition monitoring indicators include five key indicators: High-voltage explosion-proof switch ( A61) ,Explosion-proof magnetic starter(A62),Transformers(A63),etc.. 


\subsection{BP Neural Network Model Construction}

(1) Design of input layer. According to the state colliery safety monitoring equipment index evaluation system, we divided the system into six subsystems, each subsystem as a BP neural network input.So determine the BP neural network to the input layer of six; input layer node corresponds to the established system of colliery equipment in the six types of 31 indicator variables.

(2) Hidden layer design. Select one hidden layer taken for experience, its nodes $n=\operatorname{sqrt}(m+k)+a$ (a is a constant between $0 \sim 10$ ). And the node number is 17 , Through the experiment, we control the system's total error in minimum, so 17 hidden layer nodes are the best number of nodes.

(3) The output layer design. The number of output layer nodes identified as one. Device state is divided into qualified (0001) and unqualified ( 0010 ), scrapped ( 0100 )and the national disabled
(1000) .

\section{EXPERIMENTAL DESIGN}

According to the data based on the colliery equipment safety monitoring provided by 5 coal enterprises in Shanxi Province, and combined with the characteristics of colliery equipment, we extract Coal mining equipment, boring equipment, lifting equipment, drainage, ventilation and electrical equipment for six class as a predictor of BP network input layer of input.Equipment security state is divided into qualified and unqualified, scrapped and the national disabled as the four kinds of output by BP network output layer. By make the input data in normalized, we obtain the experimental data, such as in Table 1:

Table 1 Device Status predictor

\begin{tabular}{|l|c|c|c|c|c|}
\hline Predictor & Colliery 1 & Colliery 2 & Colliery 3 & Colliery 4 & Colliery 5 \\
\hline Coal mining equipment & 0.926 & 0.876 & 0.709 & 0.603 & 0.521 \\
\hline boring equipment & 0.947 & 0.913 & 0.824 & 0.725 & 0.453 \\
\hline lifting equipment & 0.918 & 0.906 & 0.836 & 0.681 & 0.436 \\
\hline drainage & 0.930 & 0.815 & 0.761 & 0.624 & 0.362 \\
\hline ventilation & 0.905 & 0.879 & 0.732 & 0.642 & 0.456 \\
\hline electrical equipment & 0.912 & 0.842 & 0.754 & 0.687 & 0.501 \\
\hline Predictions & $(0001)$ & $(0001)$ & $(0010)$ & $(0100)$ & $(1000)$ \\
\hline Device Status & qualified & qualified & unqualified & scrapped & the national disabled \\
\hline
\end{tabular}

Features in processing Non-linear problem by using neural network and the advantages Matlab owed in numerical calculations improve the accuracy and speed of prediction. This paper predicted the colliery equipment monitoring data, and using matlab toolbox for program design. By training, the network meets the requirements of the target error, Specific result shows in Figure 3 


\section{CONCLUSION}

This paper started with the point of view of a combination of theory and practice, and draw the following main conclusions by monitoring the state colliery safety equipment, as follows:

(1) BP neural network model being applied to colliery safety monitoring equipment can better the security situation on the colliery equipment prediction, and it also has a strong dynamic so as to provide decision for the manager based on a variety of views.
(2) Fault-tolerant capability. As the network of knowledge and information using a distributed storage, individual units will not cause damage to the output of the error. This not only improves the capability in forecasting and identifying fault-tolerant but also with high reliability and the value of practical and promotion.

(3) The method used in BP neural network also has some limitations. Through constant correction of the connection weights between all neurons, we achieve the global optimum. But because of the complexity of error curved surface, it is easy to make the network into a local minimum point.

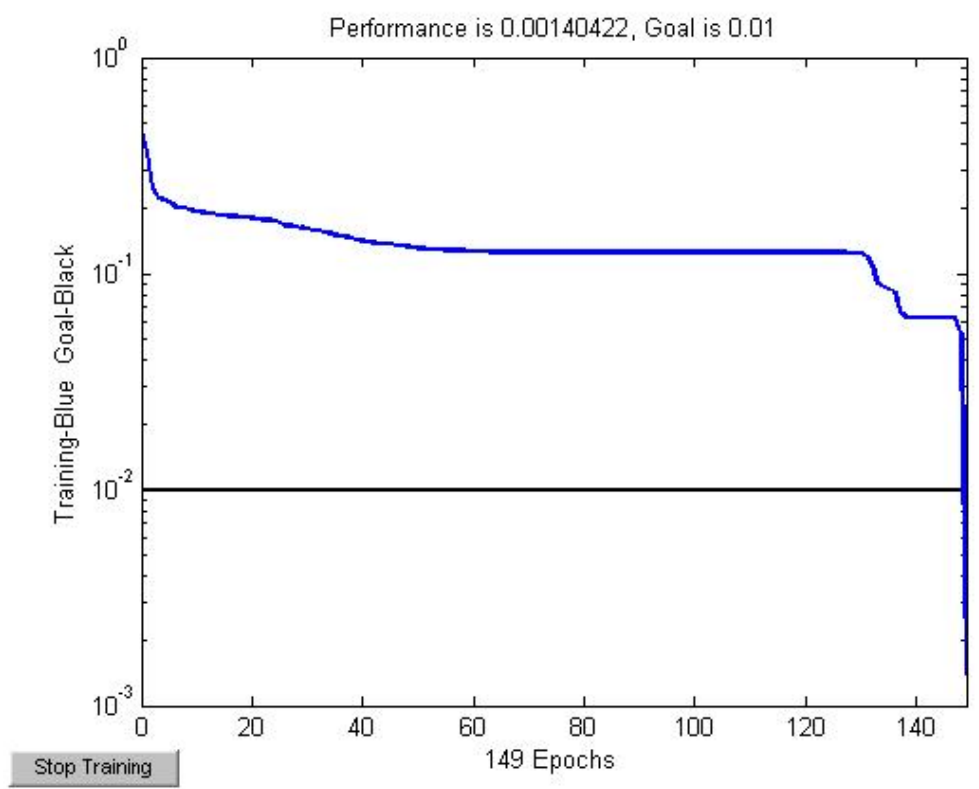

Figure 3 Simulation and Training

\section{ACKNOWLEDGEMENT}

The author would like to thank the anonymous reviewers for their valuable suggestion and comment on this work.

This thesis consists of the following three research projects to support:

1. Multi-agent artificial coal mine model modeling and the miners safety escape behavior rules study,2012011011-5

2. WEB-based safety monitoring and intelligent management system of colliery equipment , 20080321012-01

3. University youth fund:201130163.

\section{REFERENCES:}


[1] F. LiMin. An expert network for DNA sequence analysis . IEEE Intelligent Sys-tems, 1999, 65-71.

[2] Peng T M. Advancement in the Application of Neural Network for Short term Load [3] Forecasting[J]. Trans. on PW RS, 1 992(3): $250 \sim 257$.

[3] Park J M, Chong E K P'Siegel $\mathrm{H}$ ] . Efficient Multicast Packet Aut-hentication Using Signature Amortizat-ion[C]. USA. "IEEESymp Security andPri-vacy, 2002. 227-240.

[4] Perrig A. The BiBa one-time signat-ure and broadcast authentication protocol[C]. USA: 8th ACM Conf Comp and CommunSecurity,ACM,2001. 28-37.

[5] Amann P , Perronne JM, Gissinger GL.Identification of fuzzy relational models for fault detection[M] . CONTROL ENG PRACT.2001.

[6] Jon Dunsdon, Mark Harrington. The Application of Open System Ar2 chitecture for Condition BasedMaintenance to Complete IVHM [C ].2008 IEEE Aerospace Conference, 2008.

[7] Ken Cai, , Rong-qian Yang, Li-hua Li, Xiao-ming Wu, "Automatic 3D Whole Heart Registration-Based Segmentation Using Mutual Information and B-Splines", IJACT, Vol. 3, No. 11, pp. 1 8, 2011

[8] Junxi Bi, Yang Zhang, Yimin Zhang, "Design and Research of A New Kind of Latex Dipping Machine", JCIT, Vol. 7, No. 23, pp.862 869, 2012

[9] Chen Cheng, Xiaoxun Meng, "Global Manufacturing Oriented Computerized Numerical Control System Based on Embedded Opening WEB", JCIT, Vol. 7, No. 23, pp.836 844, 2012
[10] LIU Xiqing. The present conditions and development of gas monitored control system in our country coal mine [J ].Shanxi Cooking Coal Science \& Technology, 2006(3):38-40. 
Journal of Theoretical and Applied Information Technology $20^{\text {th }}$ April 2013. Vol. 50 No.2

(c) 2005 - 2013 JATIT \& LLS. All rights reserved. 